\title{
On Tannaka duality for vector bundles on $p$-adic curves
}

\author{
Christopher Deninger Annette Werner
}

Dedicated to Jacob Murre

\section{Introduction}

In our paper [DW2] we have introduced a certain category $\mathfrak{B}^{p s}$ of degree zero bundles with "potentially strongly semistable reduction" on a $p$-adic curve. For these bundles it was possible to establish a partial $p$-adic analogue of the classical NarasimhanSeshadri theory for semistable vector bundles of degree zero on compact Riemann surfaces. One of the main open questions of [DW2] was, whether our category was abelian. The first main result of the present note, Corollary [10, asserts that this is indeed the case. It follows that $\mathfrak{B}^{p s}$ is even a neutral Tannakian category. In the second main result, theorem [16, we calculate the group of connected components of the Tannaka dual group of $\mathfrak{B}^{p s}$. This uses a result of A. Weil characterizing vector bundles that become trivial in a finite étale covering as the ones satisfying a "polynomial equation" over the integers.

Bsides, in section 3 we give a short review of [DW2], and in section 2 we discuss the "strongly semistable reduction" condition.

We would like to draw the reader's attention to the paper of Faltings $[\mathrm{F}]$ on nonabelian $p$-adic Hodge theory which generalizes several results of DW2 to Higgs bundles.

It is a pleasure for us to thank Uwe Jannsen for a helpful discussion.

\section{Vector bundles in characteristic $p$}

Throughout this paper, we call a purely one-dimensional separated scheme of finite type over a field $k$ a curve over $k$.

Let $C$ be a connected smooth, projective curve over $k$. For a vector bundle $E$ on $C$ 
we denote by $\mu(E)=\frac{\operatorname{deg}(E)}{\mathrm{rk}(E)}$ the slope of $E$. Then $E$ is called semistable (respectively stable), if for all proper non-trivial subbundles $F$ of $E$ the inequality $\mu(F) \leq \mu(E)$ (respectively $\mu(F)<\mu(E)$ ) holds.

Lemma 1 If $\pi: C^{\prime} \rightarrow C$ is a finite separable morphism of connected smooth projective $k$-curves, then semistability of $E$ is equivalent to semistability of $\pi^{*} E$.

Proof See Gie2, 1.1.

If $\operatorname{char}(k)=0$, then by lemma 1 any finite morphism of smooth connected projective curves preserves semistability. However in the case $\operatorname{char}(k)=p$, there exist vector bundles which are destabilized by the Frobenius map, see [Gie1], Theorem 1. Assume that $\operatorname{char}(k)=p$, and let $F: C \rightarrow C$ be the absolute Frobenius morphism, defined by the $p$-power map on the structure sheaf.

Definition 2 A vector bundle $E$ on $C$ is called strongly semistable of degree zero if $\operatorname{deg}(E)=0$ and if $F^{n *} E$ is semistable on $C$ for all $n \geq 0$.

Now we also consider non-smooth curves over $k$. Let $Z$ be a proper curve over $k$. By $C_{1}, \ldots, C_{r}$ we denote the irreducible components of $Z$ endowed with their reduced induced structures. Let $\tilde{C}_{i}$ be the normalization of $C_{i}$, and write $\alpha_{i}: \tilde{C}_{i} \rightarrow C_{i} \rightarrow Z$ for the canonical map. Note that the curves $\tilde{C}_{i}$ are smooth irreducible and projective over $k$.

Definition 3 A vector bundle $E$ on the proper $k$-curve $Z$ is called strongly semistable of degree zero, if all $\alpha_{i}^{*} E$ are strongly semistable of degree zero.

A alternative characterization of this propery is given by the following result.

Proposition $4 A$ vector bundle $E$ on $Z$ is strongly semistable of degree zero if and only if for any $k$-morphism $\pi: C \rightarrow Z$, where $C$ is a smooth connected projective curve over $k$, the pullback $\pi^{*} E$ is semistable of degree zero on $C$.

Note that in [DM], (2.34) bundles with this property are called semistable of degree zero. 
Proof Let $X$ be a scheme over $k$. The absolute Frobenius $F$ sits in a commutative diagram

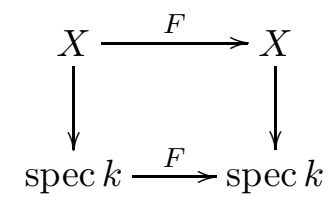

If we denote for all $r \geq 1$ by $X^{(r)}$ the scheme $X$ together with the structure map $X \rightarrow \operatorname{spec} k \stackrel{F^{r}}{\rightarrow} \operatorname{spec} k$, then $F^{r}: X^{(r)} \rightarrow X$ is a morphism over spec $k$.

Assume that the curve $Z$ has the property in the claim. Applying it to the smooth projective curves $\tilde{C}_{i}$ and the $k$-morphisms

$$
\tilde{C}_{i}^{(r)} \stackrel{F^{r}}{\longrightarrow} \tilde{C}_{i} \stackrel{\alpha_{i}}{\longrightarrow} Z
$$

we find that all $\alpha_{i}^{*} E$ are strongly semistable of degree zero, i.e. that $E$ is strongly semistable of degree zero in the sense of definition 3 .

Conversely, assume that all $\alpha_{i}^{*} E$ are strongly semistable of degree zero. Let $\pi: C \rightarrow$ $Z$ be a $k$-morphism from a smooth connected projective curve $C$ to $Z$. Then $\pi$ factors through one of the $C_{i}$. If $\pi$ is constant, then $\pi^{*} E$ is trivial, hence semistable of degree zero. Hence we can assume that $\pi(C)=C_{i}$. Since $C$ is smooth, $\pi$ also factors through the normalization $\tilde{C}_{i}$, i.e. there is a morphism $\pi_{i}: C \rightarrow \tilde{C}_{i}$ satisfying $\alpha_{i} \circ \pi_{i}=\pi$. Since $\pi$ is dominant, it is finite and hence $\pi_{i}$ is the composition of a separable map and a power of Frobenius, see e.g. Ha, IV, 2.5. Hence there exists a smooth projective curve $D$ over $k$ and a finite separable morphism $f: D \rightarrow \tilde{C}_{i}$ such that $C \stackrel{\sim}{\longrightarrow} D^{(r)}$ for some $r \geq 1$ and $\pi_{i}$ factors as

$$
\pi_{i}: C \stackrel{\sim}{\longrightarrow} D^{(r)} \stackrel{F^{r}}{\longrightarrow} D \stackrel{f}{\rightarrow} \tilde{C}_{i} .
$$

Write $E_{i}=\alpha_{i}^{*} E$. Then we have to show that $\pi^{*} E=\pi_{i}^{*} E_{i}$ is semistable of degree 0 on $C$. By assumption, $E_{i}$ is strongly semistable of degree 0 on $\tilde{C}_{i}$. Using Lemma 1 and the fact that $F$ commutes with all morphisms in characteristic $p$, we find that the pullback $f^{*} E_{i}$ under the finite, separable map $f$ is strongly semistable of degree 0 on $D$. Hence $F^{r *} f^{*} E_{i}$ is semistable, which implies that $\pi_{i}^{*} E_{i}$ is semistable of degree zero.

Generalizing a result by Lange and Stuhler in [LS], one can show

Proposition 5 If $k=\mathbb{F}_{q}$ is a finite field, then a vector bundle $E$ on the proper $k$-curve $Z$ is strongly semistable of degree zero, if and only if there exists a finite surjective morphism

$$
\varphi: Y \rightarrow Z
$$


of proper $k$-curves such that $\varphi^{*} E$ is trivial. In fact, one can take $\varphi$ to be the composition

$$
\varphi: Y \stackrel{\mathrm{Fr}_{q}^{s}}{\rightarrow} Y \stackrel{\pi}{\rightarrow} Z
$$

of a power of the $k$-linear Frobenius morphism $\mathrm{Fr}_{q}$ (defined by the q-th power map on $\left.\mathcal{O}_{Y}\right)$ and a finite, étale and surjective morphism $\pi$.

Proof See [DW2, Theorem 18.

Note that every vector bundle $E$ of degree zero on a smooth geometrically connected projective curve $C$ of genus $g \leq 1$ is semistable. Namely, for $g=0$, every vector bundle of degree zero is in fact trivial. For $g=1$, the claim follows from Atiyah's classification [At]: let $E=\bigoplus_{i} E_{i}$ be the decomposition of $E$ into indecomposable components. Since $E$ is semistable of degree zero, all $E_{i}$ have degree zero since they are subbundles and quotients. Therefore by At Theorem 5 , we have $E_{i} \simeq L \otimes G$, where $L$ is a line bundle of degree zero and $G$ is an iterated extension of trivial line bundles. The pullback of $E_{i}$ under some Frobenius power is also of this form. Since the category of semistable vector bundles of degree 0 on $C$ is closed under extensions and contains all line bundles of degree zero, we conclude that $E_{i}$ is indeed strongly semistable of degree 0 . This proves the following fact:

Lemma 6 Let $Z$ be a proper $k$-curve such that the normalizations $\tilde{C}_{i}$ of all irreducible components $C_{i}$ are geometrically connected of genus $g\left(\tilde{C}_{i}\right) \leq 1$. Consider a vector bundle $E$ on $Z$. If all restriction $\left.E\right|_{\tilde{C}_{i}}$ are semistable of degree zero, then $E$ is strongly semistable of degree zero.

By Gie1, for every genus $\geq 2$ there are examples of semistable vector bundles of degree zero which are not strongly semistable.

On the other hand, there are results indicating that there are "a lot of" strongly semistable vector bundles of degree zero. In $[\mathrm{LP}$, Laszlo and Pauly show that for an ordinary smooth projective curve $C$ of genus two over an algebraically closed field $k$ of characteristic two, the set of strongly semistable rank two bundles is Zariski dense in the coarse moduli space of all semistable rank two bundles with trivial determinant. See [JRXY] for generalizations to higher genus.

In $[\mathrm{Du}]$, Ducrohet investigates the case of a supersingular smooth projective curve of genus two over an algebraically closed field $k$ with $\operatorname{char}(k)=2$. It turns out that in this case all equivalence classes of semistable bundles with trivial determinant but one are in fact strongly semistable. 


\section{Vector bundles on $p$-adic curves}

Before discussing the $p$-adic case, let us recall some results in the complex case, i.e. regarding vector bundles on a compact Riemann surface $X$. Let $x \in X$ be a base point and denote by $\pi: \tilde{X} \rightarrow X$ the universal covering of $X$. Every representation $\rho: \pi_{1}(X, x) \rightarrow \mathrm{GL}_{r}(\mathbb{C})$ gives rise to a flat vector bundle $E_{\rho}$ on $X$, which is defined as the quotient of the trivial bundle $\tilde{X} \times \mathbb{C}^{r}$ by the $\pi_{1}(X, x)$-action given by combining the natural action of $\pi_{1}(X, x)$ on the first factor with the action induced by $\rho$ on the second factor. It is easily seen that every flat vector bundle on $X$ is isomorphic to some $E_{\rho}$. Regarding $E_{\rho}$ as a holomorphic bundle on $X$, a theorem of Weil [W] says that a holomorphic bundle $E$ on $X$ is isomorphic to some $E_{\rho}$ (i.e. $E$ comes from a representation of $\left.\pi_{1}(X, x)\right)$ if and only if in the decomposition $E=\bigoplus_{i=1}^{r} E_{i}$ of $E$ into indecomposable subbundles all $E_{i}$ have degree zero. A famous result by Narasimhan and Seshadri $[\mathrm{NS}$ says that a holomorphic vector bundle $E$ of degree 0 on $X$ is stable if and only if $E$ is isomorphic to $E_{\rho}$ for some irreducible unitary representation $\rho$. Hence a holomorphic vector bundle comes from a unitary representation $\rho$ if and only if it is of the form $E=\bigoplus_{i=1}^{r} E_{i}$ for stable (and hence indecomposable) subbundles of degree zero.

Now let us turn to the $p$-adic case. Let $X$ be a connected smooth projective curve over the algebraic closure $\overline{\mathbb{Q}}_{p}$ of $\mathbb{Q}_{p}$ and put $X_{\mathbb{C}_{p}}=X \otimes_{\overline{\mathbb{Q}}_{p}} \mathbb{C}_{p}$. We want to look at $p$-adic representations of the algebraic fundamental group $\pi_{1}(X, x)$ where $x \in X\left(\mathbb{C}_{p}\right)$ is a base point. It is defined as follows. Denote by $F_{x}$ the functor from the category of finite étale coverings $X^{\prime}$ of $X$ to the category of finite sets which maps $X^{\prime}$ to the

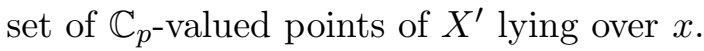

For $x, x^{\prime} \in X\left(\mathbb{C}_{p}\right)$ we call any isomorphism $F_{x} \stackrel{\sim}{\longrightarrow} F_{x^{\prime}}$ of fibre functors an étale path from $x$ to $x^{\prime}$. (Note that any topological path on a Riemann surface induces naturally such an isomorphism of fibre functors.) Then the étale fundamental group $\pi_{1}(X, x)$ is defined as

$$
\pi_{1}(X, x)=\operatorname{Aut}\left(F_{x}\right)
$$

The goal of our papers [DW1] and [DW2] is to associate $p$-adic representations of the étale fundamental group $\pi_{1}(X, x)$ to certain vector bundles on $X_{\mathbb{C}_{p}}$. Let us briefly describe the main result. We call any finitely presented, proper and flat scheme $\mathfrak{X}$ over the integral closure $\overline{\mathbb{Z}}_{p}$ of $\mathbb{Z}_{p}$ in $\overline{\mathbb{Q}}_{p}$ with generic fibre $X$ a model of $X$. By o we denote the ring of integers in $\mathbb{C}_{p}$, and by $k=\overline{\mathbb{F}}_{p}$ the residue field of $\overline{\mathbb{Z}}_{p}$ and $\mathfrak{o}$. We write $\mathfrak{X}_{\mathfrak{o}}=\mathfrak{X} \otimes_{\mathbb{Z}_{p}} \mathfrak{o}$ and $\mathfrak{X}_{k}=\mathfrak{X} \otimes_{\overline{\mathbb{Z}}_{p}} k$.

Definition 7 We say that a vector bundle $E$ on $X_{\mathbb{C}_{p}}$ has strongly semistable reduction of degree zero if $E$ is isomorphic to the generic fibre of a vector bundle $\mathcal{E}$ on $\mathfrak{X}_{\mathfrak{o}}$ 
for some model $\mathfrak{X}$ of $X$, such that the special fibre $\mathcal{E}_{k}$ is a strongly semistable vector bundle of degree zero on the proper $k$-curve $\mathfrak{X}_{k}$.

$E$ has potentially strongly semistable reduction of degree zero if there is a finite morphism $\alpha: Y \rightarrow X$ of connected smooth projective curves over $\overline{\mathbb{Q}}_{p}$ such that $\alpha_{\mathbb{C}_{p}}^{*} E$ has strongly semistable reduction of degree zero on $Y_{\mathbb{C}_{p}}$.

By $\mathfrak{B}^{s}$ (respectively $\mathfrak{B}^{p s}$ ) we denote the full subcategory of the category of vector bundles on $X_{\mathbb{C}_{p}}$ consisting of all $E$ with strongly semistable (respectively potentially strongly semistable) reduction of degree zero. Besides, for every divisor $D$ on $X_{\mathbb{C}_{p}}$ we define $\mathfrak{B}_{X_{\mathbb{C}_{p}}, D}$ to be the full subcategory of those vector bundles $E$ on $X_{\mathbb{C}_{p}}$ which can be extended to a vector bundle $\mathcal{E}$ on $\mathfrak{X}_{\mathfrak{o}}$ for some model $\mathfrak{X}$ of $X$, such that there exists a finitely presented proper $\overline{\mathbb{Z}}_{p}$-morphism

$$
\pi: \mathcal{Y} \longrightarrow \mathfrak{X}
$$

satisfying the following two properties:

i) The generic fibre of $\pi$ is finite and étale outside $D$

ii) The pullback $\pi_{k}^{*} \mathcal{E}_{k}$ of the special fibre of $\mathcal{E}$ is trivial on $\mathcal{Y}_{k}$ (c.f. [DW2, definition 6 and theorem 16).

Then we show in DW2, Theorem 17:

$$
\mathfrak{B}^{s}=\bigcup_{D} \mathfrak{B}_{X_{\mathbb{C}_{p}}, D}
$$

where $D$ runs through all divisors on $X_{\mathbb{C}_{p}}$. By [DW2], Theorem 13, every bundle in $\mathfrak{B}_{X_{\mathbb{C}_{p}, D}}$ is semistable of degree zero, so that $\mathfrak{B}^{s}$ and also $\mathfrak{B}^{p s}$ are full subcategories of the category $\mathfrak{T}^{s s}$ of semistable bundles of degree zero on $X_{\mathbb{C}_{p}}$. Line bundles of degree zero lie in $\mathfrak{B}^{p s}$ by DW2 Theorem $12 \mathrm{a}$.

The main result in DW2] is the following (c.f. DW2], theorem 36):

Theorem 8 Let $E$ be a bundle in $\mathfrak{B}^{p s}$. For every étale path from $x$ to $y$ in $X\left(\mathbb{C}_{p}\right)$ there is an isomorphism

$$
\rho_{E}(\gamma): E_{x} \stackrel{\sim}{\longrightarrow} E_{y}
$$

of "parallel transport", which behaves functorially in $\gamma$. The association $E \mapsto \rho_{E}(\gamma)$ is compatible with tensor products, duals and internal homs of vector bundles in the obvious way. It is also compatible with $\operatorname{Gal}\left(\overline{\mathbb{Q}}_{p} / \mathbb{Q}_{p}\right)$-conjugation. Besides, if $\alpha: X \rightarrow X^{\prime}$ is a morphism of smooth projective curves over $\overline{\mathbb{Q}}_{p}$ and $E^{\prime}$ a bundle in $\mathfrak{B}_{X_{\mathbb{C}_{p}}^{\prime}}^{p s}$, then $\rho_{\alpha^{*} E^{\prime}}(\gamma)$ and $\rho_{E^{\prime}}\left(\alpha_{*} \gamma\right)$ coincide, where $\alpha_{*} \gamma$ is the induced étale path on $X^{\prime}$. For every $x \in X\left(\mathbb{C}_{p}\right)$ the fibre functor

$$
\mathfrak{B}^{p s} \longrightarrow \operatorname{Vec}_{\mathbb{C}_{p}},
$$


mapping $E$ to the fibre $E_{x}$ in the category $\operatorname{Vec}_{\mathbb{C}_{p}}$ of $\mathbb{C}_{p}$-vector spaces, is faithful.

In particular one obtains a continuous representation $\rho_{E, x}: \pi_{1}(X, x) \rightarrow \mathrm{GL}_{r}\left(E_{x}\right)$. The functor $E \mapsto \rho_{E, x}$ is compatible with tensor products, duals, internal homs, pullbacks of vector bundles and $\operatorname{Gal}\left(\overline{\mathbb{Q}}_{p} / \mathbb{Q}_{p}\right)$-conjugation.

Let us look at two special cases of this representation: For line bundles on a curve $X$ with good reduction, $\rho$ induces a homomorphism

$$
\operatorname{Pic}_{X}^{0}\left(\mathbb{C}_{p}\right) \longrightarrow \operatorname{Hom}_{\text {cont }}\left(\pi_{1}(X, x), \mathbb{C}_{p}^{*}\right)
$$

mapping $L$ to $\rho_{L, x}$. As shown in DW1 this map coincides with the map defined by Tate in Ta $\S 4$ on an open subgroup of $\operatorname{Pic}_{X}^{0}\left(\mathbb{C}_{p}\right)$. Secondly, applying $\rho$ to bundles $E$ in $H^{1}\left(X_{\mathbb{C}_{p}}, \mathcal{O}\right)=\operatorname{Ext}_{X_{\mathbb{C}_{p}}}^{1}(\mathcal{O}, \mathcal{O})$, one recovers the Hodge-Tate map to $H^{1}\left(X_{\text {ét }}, \mathbb{Q}_{p}\right) \otimes \mathbb{C}_{p}=\operatorname{Ext}_{\pi_{1}(X, x)}^{1}\left(\mathbb{C}_{p}, \mathbb{C}_{p}\right)$, see DW1], corollary 8.

It follows from DW2, Proposition 9 and Theorem 11 that the categories $\mathfrak{B}^{s}$ and $\mathfrak{B}^{p s}$ are closed under tensor products, duals, internal homs and extensions. We will now prove another important property of those categories.

Theorem 9 If a vector bundle $E$ on $X_{\mathbb{C}_{p}}$ is contained in $\mathfrak{B}^{s}$ (respectively $\mathfrak{B}^{p s}$ ), then every quotient bundle of degree zero and every subbundle of degree zero of $E$ is also contained in $\mathfrak{B}^{s}$ (respectively $\mathfrak{B}^{p s}$ ).

Proof It suffices to show this property for the category $\mathfrak{B}^{s}$. By duality, it suffices to treat quotient bundles. So let $\tilde{\mathcal{E}}$ be a vector bundle with strongly semistable reduction of degree zero on $\mathfrak{X}_{\mathfrak{o}}$, where $\mathfrak{X}$ is a model of $X$. Denote by $E$ the generic fibre of $\tilde{\mathcal{E}}$, and let

$$
0 \rightarrow E^{\prime} \rightarrow E \rightarrow E^{\prime \prime} \rightarrow 0
$$

be an exact sequence of vector bundles $X_{\mathbb{C}_{p}}$, where $E^{\prime \prime}$ has degree zero. By DW2, Theorem $5, E^{\prime}$ can be extended to a vector bundle $\mathcal{F}^{\prime}$ on $\mathcal{Y}_{\mathfrak{o}}$, where $\mathcal{Y}$ is a model of $X$ such that there is a morphism $\varphi: \mathcal{Y} \rightarrow \mathfrak{X}$ inducing an isomorphism on the generic fibres. Since $\operatorname{Hom}\left(\mathcal{F}^{\prime}, \varphi_{\mathfrak{o}}^{*} \tilde{\mathcal{E}}\right) \otimes_{\mathfrak{o}} \mathbb{C}_{p}=\operatorname{Hom}\left(E^{\prime}, E\right)$, we may assume that the embedding $E^{\prime} \rightarrow E$ can be extended to a $\mathcal{O}_{\mathcal{Y}_{\mathfrak{0}}}$-module homomorphism $\mathcal{F}^{\prime} \rightarrow \varphi_{\mathfrak{0}}^{*} \tilde{\mathcal{E}}$ after changing the morphisms in the diagram (11).

Let $\mathcal{F}^{\prime \prime}$ be the quasi-coherent sheaf on $\mathcal{Y}_{\mathfrak{o}}$ such that $\mathcal{F}^{\prime} \rightarrow \varphi_{\mathfrak{o}}^{*} \tilde{\mathcal{E}} \rightarrow \mathcal{F}^{\prime \prime} \rightarrow 0$ is exact. Then $\mathcal{F}^{\prime \prime}$ is of finite presentation. Note that the generic fibre of this sequence is isomorphic to the sequence (11). 
Let $r$ be the rank of $E^{\prime \prime}$. The same argument as in the proof of [DW2], Theorem 5 shows that the blowing-up

$$
\psi_{\mathfrak{o}}: \mathfrak{Z}_{\mathfrak{o}} \rightarrow \mathcal{Y}_{\mathfrak{o}}
$$

of the $r$-th Fitting ideal of $\mathcal{F}^{\prime \prime}$ descends to a finitely presented morphism $\psi: \mathfrak{Z} \rightarrow \mathcal{Y}$ inducing an isomorphism on the generic fibres. Besides, if $\mathcal{A}$ denotes the annihilator of the $r$-th Fitting ideal of $\psi_{\mathfrak{o}}^{*} \mathcal{F}^{\prime \prime}$, the sheaf $\psi_{\mathfrak{o}}^{*} \mathcal{F}^{\prime \prime} / \mathcal{A}$ is locally free by [RG] (5.4.3). Hence it gives rise to a vector bundle $\mathcal{E}^{\prime \prime}$ on $\mathfrak{Z}_{\mathfrak{o}}$ with generic fibre $E^{\prime \prime}$. Let us write $\mathcal{E}=\psi_{\mathrm{o}}^{*} \varphi_{\mathrm{o}}^{*} \tilde{\mathcal{E}}$. Then we have a natural surjective homomorphism of vector bundles $\mathcal{E} \rightarrow \mathcal{E}^{\prime \prime}$ on $\mathfrak{Z}_{\mathfrak{o}}$ extending the quotient map $E \rightarrow E^{\prime \prime}$ on the generic fibre $X_{\mathbb{C}_{p}}$.

Let $K$ be a finite extension of $\mathbb{Q}_{p}$ such that $\mathfrak{Z}$ descends to a proper and flat scheme $\mathfrak{Z}_{\mathfrak{o}_{K}}$ over the ring of integers $\mathfrak{o}_{K}$. We can choose $K$ big enough so that all irreducible components of the special fibre of $\mathfrak{Z}$ are defined over the residue field of $K$. Let $X_{K}$ be the generic fibre of $\mathfrak{Z}_{\mathfrak{o}_{K}}$. Then $X_{K} \otimes_{K} \overline{\mathbb{Q}}_{p} \simeq X$. The scheme $\mathfrak{Z}_{\mathfrak{o}}$ is the projective limit of all $\mathfrak{Z}_{A}=\mathfrak{Z}_{\mathfrak{o}_{K}} \otimes_{\mathfrak{o}_{K}} A$, where $A$ runs over the finitely generated $\mathfrak{o}_{K}$-subalgebras of $\mathfrak{o}$.

By [EGAIV], (8.5.2), (8.5.5), (8.5.7), (11.2.6) there exists a finitely generated $\mathfrak{o}_{K^{-}}$ subalgebra $A$ of $\mathfrak{o}$ with quotient field $Q \subset \mathbb{C}_{p}$ such that $\mathcal{E} \rightarrow \mathcal{E}^{\prime \prime}$ descends to a surjective homomorphism $\mathcal{E}_{A} \rightarrow \mathcal{E}_{A}^{\prime \prime}$ of vector bundles on $\mathfrak{Z}_{A}$.

Let $x \in \operatorname{spec} A$ be the point corresponding to the prime ideal $A \cap \mathfrak{m}$ in $A$, where $\mathfrak{m} \subset \mathfrak{o}$ is the valuation ideal. If $\pi_{K}$ is a prime element in $\mathfrak{o}_{K}$, we have $\mathfrak{o}_{K} /\left(\pi_{K}\right) \subset$ $A / A \cap \mathfrak{m} \subset \mathfrak{o} / \mathfrak{m}=k$, so that $A \cap \mathfrak{m}$ is a maximal ideal in $A$. Hence $x$ is a closed point with residue field $\kappa=\kappa(x)$ which is a finite extension of $\mathfrak{o}_{K} /\left(\pi_{K}\right)$ in $k$.

By assumption, the vector bundle $\tilde{\mathcal{E}}_{k}$ on the special fibre $\mathfrak{X}_{k}$ of $\mathfrak{X}$ is strongly semistable of degree zero. By Proposition 4 strong semistability is preserved under pullbacks via $k$-morphisms, so that $\mathcal{E}_{k}=(\varphi \circ \psi)_{k}^{*} \tilde{\mathcal{E}}_{k}$ is also strongly semistable of degree zero.

The bundle $\mathcal{E}_{\kappa}=\mathcal{E}_{A} \otimes_{A} \kappa$ satisfies $\mathcal{E}_{\kappa} \otimes_{\kappa} k \simeq \mathcal{E}_{k}$, hence it is strongly semistable of degree zero on $\mathfrak{Z}_{\kappa}=\mathfrak{Z}_{A} \otimes_{A} \kappa$.

Let $C_{1}, \ldots, C_{r}$ be the irreducible components of $\mathfrak{Z}_{\kappa}$ with normalizations $\tilde{C}_{1}, \ldots, \tilde{C}_{r}$ and denote by $\alpha_{i}: \tilde{C}_{i} \rightarrow C_{i} \rightarrow \mathfrak{Z}_{\kappa}$ the natural map. Since the Euler characteristics are locally constant in the fibres of the flat and proper $A$-scheme $\mathfrak{Z}_{A}$, we find $\operatorname{deg} \mathcal{E}_{\kappa}^{\prime \prime}=$ $\operatorname{deg} E_{Q}^{\prime \prime}=0$.

By the degree formula in [BLR], 9.1, Proposition $5, \operatorname{deg}\left(\mathcal{E}_{\kappa}^{\prime \prime}\right)$ is a linear combination of the $\operatorname{deg}\left(\alpha_{i}^{*} \mathcal{E}_{\kappa}^{\prime \prime}\right)$ 's with positive coefficients. Since $\alpha_{i}^{*} \mathcal{E}_{\kappa}^{\prime \prime}$ is a quotient bundle of the semistable degree zero vector bundle $\alpha_{i}^{*} \mathcal{E}_{\kappa}$ on $\tilde{C}_{i}$, it has degree $\geq 0$. Hence for all $i$ we find $\operatorname{deg}\left(\alpha_{i}^{*} \mathcal{E}_{\kappa}^{\prime \prime}\right)=0$.

Now let $\mathcal{F}$ be a vector bundle on the smooth projective curve $\tilde{C}_{i}$ which is a quotient 
of $\alpha_{i}^{*} \mathcal{E}_{\kappa}^{\prime \prime}$. Then $\mathcal{F}$ is also a quotient of the semistable degree zero bundle $\alpha_{i}^{*} \mathcal{E}_{\kappa}$, which implies $\operatorname{deg}(\mathcal{F}) \geq \operatorname{deg} \alpha_{i}^{*} \mathcal{E}_{\kappa}=0$. This shows that $\alpha_{i}^{*} \mathcal{E}_{\kappa}^{\prime \prime}$ is semistable of degree 0 on $\tilde{C}_{i}$. The same argument applies to all Frobenius pullbacks of $\alpha_{i}^{*} \mathcal{E}_{\kappa}^{\prime \prime}$, so that $\alpha_{i}^{*} \mathcal{E}_{\kappa}^{\prime \prime}$ is strongly semistable of degree 0 . Hence $\mathcal{E}_{\kappa}^{\prime \prime}$ is strongly semistable of degree zero on $\mathcal{Z}_{\kappa}$. By [HL], 1.3 .8 the base change $\mathcal{E}_{k}^{\prime \prime}=\mathcal{E}_{\kappa}^{\prime \prime} \otimes_{\kappa} k$ is also strongly semistable of degree zero. Since $\mathcal{E}^{\prime \prime}$ has generic fibre $E^{\prime \prime}$, it follows that $E^{\prime \prime}$ is indeed contained in $\mathfrak{B}^{s}$.

Corollary $10 \mathfrak{B}^{s}$ and $\mathfrak{B}^{\text {ps }}$ are abelian categories.

Proof Recall that $\mathfrak{B}^{s}$ and $\mathfrak{B}^{p s}$ are full subcategories of the abelian category $\mathcal{T}^{s s}$ of semistable vector bundles of degree zero on $X_{\mathbb{C}_{p}}$. Since the trivial bundle is contained in $\mathfrak{B}^{s}$ and $\mathfrak{B}^{p s}$, and both categories are closed under direct sums by [DW1, Proposition 9, they are additive.

By the theorem, $\mathfrak{B}^{s}$ and $\mathfrak{B}^{p s}$ are also closed under kernels and cokernels, hence they are abelian categories.

\section{Tannakian categories of vector bundles}

In this section we look at several categories of semistable vector bundles from a Tannakian point of view. Useful references in this context are [DM] and [S] for example.

As before let $X$ be a smooth projective curve over $\overline{\mathbb{Q}}_{p}$ with a base point $x \in X\left(\mathbb{C}_{p}\right)$. We call a vector bundle on $X_{\mathbb{C}_{p}}$ polystable of degree zero if it is isomorphic to the direct sum of stable vector bundles of degree zero. Let $\mathcal{T}_{\text {red }}^{\text {ss }}$ be the strictly full subcategory of vector bundles on $X_{\mathbb{C}_{p}}$ consisting of polystable bundles of degree zero and set $\mathfrak{B}_{\text {red }}^{p s}=\mathfrak{B}^{p s} \cap \mathcal{T}_{\text {red }}^{s s}$. Then we have the following diagram of fully faithful embeddings

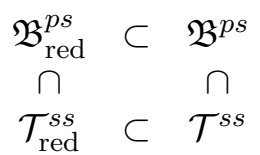

Note that because of theorem 9 , every vector bundle $E$ in $\mathfrak{B}_{\text {red }}^{\text {ps }}$ is the direct sum of stable vector bundles of degree zero contained in $\mathfrak{B}^{p s}$.

Lemma 11 The categories $\mathcal{T}_{\text {red }}^{\text {ss }}$ and $\mathfrak{B}_{\text {red }}^{\text {ps }}$ are closed under taking subquotients in $\mathcal{T}^{s s}$. 
Proof Since $\mathfrak{B}^{p s}$ is closed under subquotients in $\mathcal{T}^{s s}$ by theorem 9 it suffices to consider $\mathcal{T}_{\text {red }}^{s s}$. A bundle $E$ in $\mathcal{T}_{\text {red }}^{s s}$ can be written as $E=\bigoplus_{i} E_{i}$ with $E_{i}$ stable of degree zero. Let $\varphi: E \rightarrow E^{\prime \prime}$ be a surjective map in $\mathcal{T}^{s s}$. Then the images $\varphi\left(E_{i}\right)$ lie in $\mathcal{T}^{s s}$ and the surjective map $\left.\varphi\right|_{E_{i}}: E_{i} \rightarrow \varphi\left(E_{i}\right)$ is an isomorphism or the zero map since $E_{i}$ is stable. Hence we have $E^{\prime \prime}=\sum_{j} E_{j}^{\prime \prime}$ for stable degree zero vector bundles $E_{j}^{\prime \prime}$ (the nonzero $\varphi\left(E_{i}\right)$ ). For every $j$ we have

$$
E_{j}^{\prime \prime} \cap \sum_{k \neq j} E_{k}^{\prime \prime}=E_{j}^{\prime \prime} \quad \text { or }=0
$$

since the bundle $E_{j}^{\prime \prime}$ being stable is a simple object of $\mathcal{T}_{s s}$. It follows that $E^{\prime \prime}$ is the direct sum of suitably chosen $E_{j}^{\prime \prime}$ 's and hence lies in $\mathcal{T}_{\text {red }}^{s s}$. The case of subobjects follows by duality.

Consider the fibre functor $\omega_{x}$ on $\mathcal{T}^{s s}$ defined by $\omega_{x}(E)=E_{x}$ and $\omega_{x}(f)=f_{x}$. It induces fibre functors on the other categories as well.

Theorem 12 a The categories $\mathfrak{B}_{\text {red }}^{p s}, \mathfrak{B}^{p s}, \mathcal{T}_{\text {red }}^{s s}$ and $\mathcal{T}^{s s}$ with the fibre functor $\omega_{x}$ are neutral Tannakian categories over $\mathbb{C}_{p}$.

b The categories $\mathfrak{B}_{\text {red }}^{p s}$ and $\mathcal{T}_{\text {red }}^{\text {ss }}$ are semisimple. Every object in $\mathfrak{B}^{p s}$ (resp. $\mathcal{T}^{s s}$ ) is a successive extension of objects of $\mathfrak{B}_{\text {red }}^{\text {ps }}$ (resp. $\left.\mathcal{T}_{\text {red }}^{\text {ss }}\right)$.

c The natural inclusion $\mathfrak{B}^{p s} \subset \mathcal{T}^{s s}$ is an equivalence of categories if and only if $\mathfrak{B}_{\text {red }}^{\text {ps }} \subset \mathcal{T}_{\text {red }}^{\text {ss }}$ is an equivalence of categories.

Proof a For $\mathcal{T}^{s s}$ and $\mathcal{T}_{\text {red }}^{s s}$ this is well known, see e.g. [Si], p. 29. The categories $\mathfrak{B}^{p s}$ and $\mathfrak{B}_{\text {red }}^{p s}$ are abelian by corollary [10] and lemma 11. It was shown in [DW2] that $\mathfrak{B}^{p s}$ is closed under tensor products and duals. The same follows for $\mathfrak{B}_{\text {red }}^{p s}=$ $\mathfrak{B}^{p s} \cap \mathcal{T}_{\text {red }}^{s s}$. Faithfulness of $\omega_{x}$ on $\mathfrak{B}^{p s}$ and $\mathfrak{B}_{\text {red }}^{p s}$ follows because $\omega_{x}$ is faithful on $\mathcal{T}^{s s}$. Alternatively a direct proof was given in DW2 Theorem 36.

b Every object in $\mathfrak{B}_{\text {red }}^{p s}$ and $\mathcal{T}_{\text {red }}^{\text {ss }}$ is the direct sum of simple objects since stable bundles are simple. It is well known that objects of $\mathcal{T}^{s s}$ are successive extensions of stable bundles of degree zero. Since subquotients in $\mathcal{T}^{s s}$ of objects in $\mathfrak{B}^{p s}$ lie in $\mathfrak{B}^{p s}$ by theorem 9 , the corresponding assertion for $\mathfrak{B}^{p s}$ follows.

c This is a consequence of $\mathbf{b}$ because both $\mathcal{T}^{s s}$ and $\mathfrak{B}^{p s}$ are closed under extensions, c.f. DW2].

Let

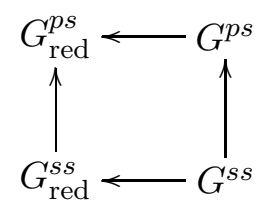


be the diagram of affine group schemes over $\mathbb{C}_{p}$ corresponding to diagram (2) by Tannakian duality.

Proposition 13 All morphisms in (3) are faithfully flat. The connected components of $G_{\mathrm{red}}^{\text {ps }}$ and $G_{\mathrm{red}}^{\text {ss }}$ are pro-reductive.

Proof The following is known DM] Proposition 2.21:

A fully faithful $\otimes$-functor $F: \mathcal{C} \rightarrow \mathcal{D}$ of neutral Tannakian categories over a field $k$ of characteristic zero induces a faithfully flat morphism $F^{*}: G_{\mathcal{D}} \rightarrow G_{\mathcal{C}}$ of the Tannakian duals if and only if we have: Every subobject in $\mathcal{D}$ of an object $F(C)$ for some $C$ in $\mathcal{C}$ is isomorphic to $F\left(C^{\prime}\right)$ for a subobject $C^{\prime}$ of $C$.

This criterion can be verified immediately for the functors in (2) by using either theorem 9 or lemma 11. The second assertion of the proposition follows from DM] Proposition 2.23 and Remark 2.28 .

Let $\mathcal{T}_{\text {fin }}$ be the category of vector bundles on $X_{\mathbb{C}_{p}}$ which are trivialized by a finite étale covering of $X_{\mathbb{C}_{p}}$. Noting that $\pi_{1}\left(X_{\mathbb{C}_{p}}, x\right) \stackrel{\sim}{\longrightarrow} \pi_{1}(X, x)$ is an isomorphism it follows that $\mathcal{T}_{\text {fin }}$ is equivalent to the category of representations of $\pi_{1}(X, x)$ with open kernels on finite dimensional $\mathbb{C}_{p}$-vector spaces $V$, c.f. [LS], 1.2. Such a representation factors over a finite quotient $G$ of $\pi_{1}(X, x)$ and the corresponding bundle in $\mathcal{T}_{\text {fin }}$ is $E=X_{\mathbb{C}_{p}}^{\prime} \times^{G} \mathbf{V}$. Here $\alpha: X^{\prime} \rightarrow X$ is the Galois covering corresponding to the quotient $\pi_{1}(X, x) \rightarrow G$ and $\mathbf{V}$ is the affine space over $\mathbb{C}_{p}$ corresponding to $V$. With the fibre functor $\omega_{x}$ the category $\mathcal{T}_{\text {fin }}$ is neutral Tannakian over $\mathbb{C}_{p}$ with Tannaka dual

$$
\pi_{1}(X, x) / \mathbb{C}_{p}=\lim _{N}\left(\pi_{1}(X, x) / N\right) / \mathbb{C}_{p}
$$

Here $N$ runs over the open normal subgroups of $\pi_{1}(X, x)$ and for a finite (abstract) group $H$ we denote by $H_{/ \mathbb{C}_{p}}$ the corresponding constant group scheme. Using Maschke's theorem it follows that $\mathcal{T}_{\text {fin }}$ is semisimple.

Proposition 14 The category $\mathcal{T}_{\text {fin }}$ is a full subcategory of $\mathfrak{B}_{\text {red }}^{\text {ps }}$. The induced morphism $G_{\text {red }}^{p s} \rightarrow \pi_{1}(X, x)_{/ \mathbb{C}_{p}}$ is faithfully flat.

Proof Using DW2 Proposition 37, one sees that $\mathcal{T}_{\text {fin }}$ is a full subcategory of $\mathfrak{B}^{p s}$. If $V$ is a finite dimensional $\mathbb{C}_{p}$-vector space on which $\pi_{1}(X, x)$ acts via a finite quotient, we can decompose $V$ into a direct sum $V=\bigoplus_{i} V_{i}$ of irreducible representations using Maschke's theorem. Using an algebraic isomorphism of $\mathbb{C}_{p}$ with $\mathbb{C}$ and NarasimhanSeshadri theory it follows that the degree zero vector bundle $E_{i}=X_{\mathbb{C}_{p}}^{\prime} \times^{G} \mathbf{V}_{i}$ is stable. Hence $E=\bigoplus E_{i}$ is an object of $\mathcal{T}_{\text {red }}^{s s}$ and hence of $\mathfrak{B}_{\text {red }}^{p s}=\mathfrak{B}^{p s} \cap \mathcal{T}_{\text {red }}^{s s}$. The 
next assertion follows from fully faithfullness of $\mathcal{T}_{\text {fin }} \hookrightarrow \mathfrak{B}_{\text {red }}^{p s}$ since $\mathfrak{B}_{\text {red }}^{p s}$ is semisimple, c.f. [DM], Remark 2.29.

Consider a Galois covering $\alpha: X^{\prime} \rightarrow X$ with group $\operatorname{Gal}\left(X^{\prime} / X\right)$ of smooth projective curves over $\overline{\mathbb{Q}}_{p}$ and choose a point $x^{\prime} \in X^{\prime}\left(\mathbb{C}_{p}\right)$ above $x \in X\left(\mathbb{C}_{p}\right)$. Let us write $\mathcal{C}_{X}$ for any of the categories $\mathfrak{B}_{\text {red }}^{p s}, \mathfrak{B}^{p s}, \mathcal{T}_{\text {red }}^{s s}$ and $\mathcal{T}^{s s}$ of vector bundles on $X_{\mathbb{C}_{p}}$. The pullback functor $\alpha^{*}: \mathcal{C}_{X} \rightarrow \mathcal{C}_{X^{\prime}}$ is a morphism of neutral Tannakian categories over $\mathbb{C}_{p}$ commuting with the fibre functors $\omega_{x}$ and $\omega_{x^{\prime}}$. Let $i: G_{X^{\prime}} \rightarrow G_{X}$ be the morphism of Tannaka duals induced by $\alpha^{*}$. We also need the faithfully flat homomorphism obtained by composition:

$$
q: G_{X} \rightarrow \pi_{1}(X, x) / \mathbb{C}_{p} \rightarrow \operatorname{Gal}\left(X^{\prime} / X\right) / \mathbb{C}_{p}
$$

Here the second arrow is determined by our choice of $x^{\prime}$. Note that every $\sigma$ in $\operatorname{Gal}\left(X^{\prime} / X\right)$ induces an automorphism $\sigma^{*}$ of $\mathcal{C}_{X^{\prime}}$ and hence an automorphism $\sigma$ : $G_{X^{\prime}} \rightarrow G_{X^{\prime}}$ of group schemes over $\mathbb{C}_{p}$.

Lemma 15 There is a natural exact sequence of affine group schemes over $\mathbb{C}_{p}$

$$
1 \rightarrow G_{X^{\prime}} \stackrel{i}{\rightarrow} G_{X} \stackrel{q}{\rightarrow} \operatorname{Gal}\left(X^{\prime} / X\right) / \mathbb{C}_{p} \rightarrow 1
$$

Proof Every bundle $E^{\prime}$ in $\mathcal{C}_{X^{\prime}}$ is isomorphic to a subquotient of $\alpha^{*}(E)$ for some bundle $E$ in $\mathcal{C}_{X}$. Namely, thinking of $E^{\prime}$ as a locally free sheaf, the sheaf $E=\alpha_{*} E^{\prime}$ is locally free again and we have $\alpha^{*} E \cong \bigoplus_{\sigma} \sigma^{*} E^{\prime}$ where $\sigma$ runs over $\operatorname{Gal}\left(X^{\prime} / X\right)$. Incidentally, $E$ lies in $\mathcal{C}_{X}$ because $\alpha^{*} E \cong \bigoplus_{\sigma} \sigma^{*} E^{\prime}$ lies in $\mathcal{C}_{X^{\prime}}$. This is clear for $\mathcal{C}=\mathcal{T}^{s s}$ or $\mathfrak{B}^{p s}$. For $\mathcal{T}_{\text {red }}^{s s}$ and hence for $\mathfrak{B}_{\text {red }}^{\text {ps }}$ it follows from [HL] Lemma 3.2.3.

It follows from DM Proposition 2.21 (b) that $i$ is a closed immersion. By descent the category $\mathcal{C}_{X}$ is equivalent to the category of bundles in $\mathcal{C}_{X^{\prime}}$ equipped with a $\operatorname{Gal}\left(X^{\prime} / X\right)$-operation covering the one on $X^{\prime}$. In other words, the category of representations of $G_{X}$ is equivalent to the category of representations of $G_{X^{\prime}}$ together with a $\operatorname{Gal}\left(X^{\prime} / X\right)$-action, i.e. a transitive system of isomorphisms $\sigma^{*} \rho=\rho \circ \sigma \rightarrow \rho$ for all $\sigma$ in $\operatorname{Gal}\left(X^{\prime} / X\right)$. Hence, $G_{X}$ is an extension of $G_{X^{\prime}}$ by $\operatorname{Gal}\left(X^{\prime} / X\right) / \mathbb{C}_{p}$ inducing the above $\operatorname{Gal}\left(X^{\prime} / X\right)$-action on $G_{X^{\prime}}$. (Because such an extension has the same $\otimes$-category of representations.) In particular, the sequence in the lemma is exact.

For a commutative diagram of Galois coverings

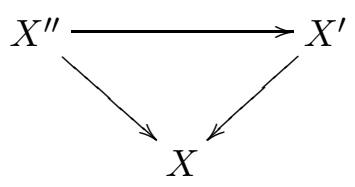


and the choice of points $x^{\prime} \in X^{\prime}\left(\mathbb{C}_{p}\right)$ and $x^{\prime \prime} \in X^{\prime \prime}\left(\mathbb{C}_{p}\right)$ over $x$ we get a commutative diagram of affine group schemes over $\mathbb{C}_{p}$ :

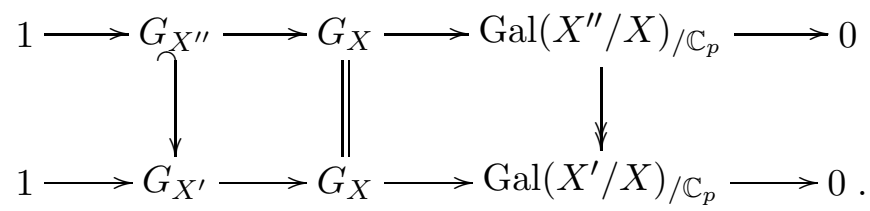

Passing to the projective limit, we get an exact sequence

$$
1 \longrightarrow \lim _{X^{\prime}} G_{X^{\prime}} \longrightarrow G_{X} \longrightarrow \pi_{1}(X, x) / \mathbb{C}_{p} \longrightarrow 1
$$

Right exactness follows from propositions 13 and 14

Theorem 16 We have a commutative diagram

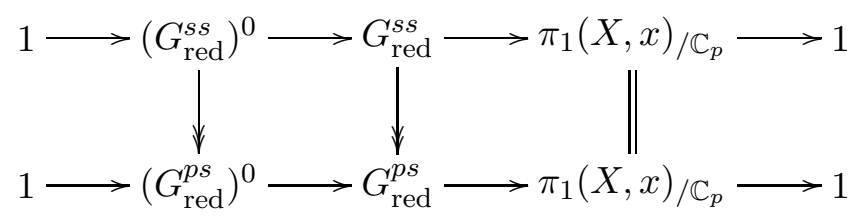

In particular $\pi_{1}(X, x) / \mathbb{C}_{p}$ is the common group scheme of connected components of both $G_{\text {red }}^{\text {ss }}$ and $G_{\text {red }}^{\text {ps }}$. Moreover we have:

$$
\left(G_{\text {red }}^{s s}\right)^{0}=\lim _{\overleftarrow{X^{\prime}}} G_{\text {red, } X^{\prime}}^{s s} \quad \text { and } \quad\left(G_{\text {red }}^{p s}\right)^{0}=\lim _{\overleftarrow{X^{\prime}}} G_{\text {red, } X^{\prime}}^{p s}
$$

Here $X^{\prime} / X$ runs over a cofinal system of pointed Galois covers of $(X, x)$.

Proof Let $\mathcal{C}_{X}$ denote either $\mathfrak{B}_{\text {red, } X}^{p s}$ or $\mathcal{T}_{\text {red, } X}^{s s}$ and let $G_{X}$ be its Tannaka dual. The exact sequence (4) implies that $G_{X}^{0} \subset \lim _{X^{\prime}} G_{X^{\prime}}$. Hence it suffices to show that $\lim _{X^{\prime}} G_{X^{\prime}}$ is connected. The category of finite dimensional representations of

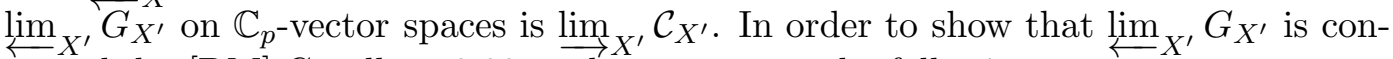
nected, by $\mathrm{DM}$ ] Corollary 2.22 we have to prove the following:

Claim Let $A$ be an object of $\lim _{X^{\prime}} \mathcal{C}_{X^{\prime}}$. Then the strictly full subcategory $[[A]]$ of $\lim _{X^{\prime}} \mathcal{C}_{X^{\prime}}$ whose objects are isomorphic to subquotients of $A^{N}, N \geq 0$ is not stable under $\otimes$ unless $A$ is isomorphic to a trivial bundle.

Proof Let $[[A]]$ be stable under $\otimes$. The category $\lim _{X^{\prime}} \mathcal{C}_{X^{\prime}}$ is semisimple since $\left(\lim _{X^{\prime}} G_{X^{\prime}}\right)^{0}=\lim _{X_{X^{\prime}}} G_{X^{\prime}}^{0}$ is pro-reductive. Hence we may decompose $A$ into simple objects $A=A_{1} \oplus \ldots \oplus A_{s}$. By assumption, for every $j \geq 1$ the object $A_{1}^{\otimes j}$ is 
isomorphic to a subquotient of $A^{N}$ for some $N=N(j)$. The same argument as in the proof of Lemma[11 shows that up to isomorphism the subquotients of $N A:=A^{N}$ have the form $m_{1} A_{1} \oplus \ldots \oplus m_{s} A_{s}$ for integers $m_{i} \geq 0$. Hence we get isomorphisms where $\sum$ means "direct sum":

$$
A_{1}^{\otimes j} \cong \sum_{i=1}^{s} m_{i j} A_{i} \quad \text { for } 1 \leq j \leq r .
$$

Here $M=\left(m_{i j}\right)$ is an $s \times r$-matrix over $\mathbb{Z}$. Fixing some $r>s$ there is a relation with integers $c_{j}$, not all zero:

$$
\sum_{j=1}^{r} c_{j}\left(m_{1 j}, \ldots, m_{s j}\right)^{t}=0
$$

This gives the relation

$$
\sum_{j=1}^{r} c_{j}^{+}\left(m_{1 j}, \ldots, m_{s j}\right)^{t}=\sum_{j=1}^{r} c_{j}^{-}\left(m_{1 j}, \ldots, m_{s j}\right)^{t}
$$

where $c_{j}^{+}=\max \left\{c_{j}, 0\right\}$ and $c_{j}^{-}=-\min \left\{c_{j}, 0\right\}$. "Left multiplication" with $\left(A_{1}, \ldots, A_{s}\right)$ gives isomorphisms

$$
\sum_{j=1}^{r} c_{j}^{+} \sum_{i=1}^{s} m_{i j} A_{i} \cong \sum_{j=1}^{r} c_{j}^{-} \sum_{i=1}^{s} m_{i j} A_{i},
$$

and hence

$$
\sum_{j=1}^{r} c_{j}^{+} A_{1}^{\otimes j} \cong \sum_{j=1}^{r} c_{j}^{-} A_{1}^{\otimes j} .
$$

For the polynomials $P^{ \pm}(T)=\sum_{j=1}^{r} c_{j}^{ \pm} T^{j}$ with coefficients in $\mathbb{Z}^{\geq 0}$ we have $P^{+} \neq P^{-}$ and:

$$
P^{+}\left(A_{1}\right) \cong P^{-}\left(A_{1}\right) \text {. }
$$

Let $E_{1}$ be a bundle in $\mathcal{C}_{X^{\prime}}$ representing $A_{1}$ in $\underline{\lim }_{X^{\prime}} \mathcal{C}_{X^{\prime}}$. Then we have an isomorphism

$$
P^{+}\left(\beta^{*} E_{1}\right) \cong P^{-}\left(\beta^{*} E_{1}\right)
$$

of vector bundles on a suitable Galois cover $\beta: X^{\prime \prime} \rightarrow X^{\prime}$. A theorem of Weil, c.f. W] Ch. III or $\left[\mathbb{N}\right.$, now implies that $\beta^{*} E_{1}$ and hence $E_{1}$ is trivialized by a finite étale covering of $X^{\prime}$. Hence $A_{1}$, the class of $E_{1}$ is isomorphic in $\lim _{X^{\prime}} \mathcal{C}_{X^{\prime}}$ to a trivial bundle. The same argument applies to $A_{2}, A_{3}, \ldots$ Hence $A$ is isomorphic to a trivial bundle as well. This proves the claim and hence the theorem. 
We now determine the structure of $G^{\text {ab }}$ for $G=G_{\text {red }}^{p s}$ and $G=G_{\text {red }}^{s s}$. This group is pro-reductive and abelian, hence diagonalizable and therefore determined by its character group

$$
X\left(G^{\mathrm{ab}}\right)=\operatorname{Mor}_{\mathbb{C}_{p}}\left(G^{\mathrm{ab}}, \mathbb{G}_{m}\right) .
$$

The characters of $G^{\mathrm{ab}}$ correspond to isomorphism classes of one-dimensional representations of $G$ i.e. to isomorphism classes of degree zero line bundles in $\mathfrak{B}_{\text {red }}^{p s}$ resp. $\mathcal{T}_{\text {red }}^{s s}$. Since both categories contain all degree zero line bundles we get

$$
X\left(G^{\mathrm{ab}}\right)=\operatorname{Pic}_{X}^{0}\left(\mathbb{C}_{p}\right)
$$

and hence

$$
\begin{aligned}
G^{\mathrm{ab}} & =\operatorname{Hom}\left(\operatorname{Pic}_{X}^{0}\left(\mathbb{C}_{p}\right), \mathbb{G}_{m, \mathbb{C}_{p}}\right) \\
& =\varliminf_{A}^{\lim } \operatorname{Hom}\left(A, \mathbb{G}_{m}, \mathbb{C}_{p}\right) .
\end{aligned}
$$

Here $A$ runs over the finitely generated subgroups of $\operatorname{Pic}_{X}^{0}\left(\mathbb{C}_{p}\right)$. A similar argument using the fact that $\left(G_{\text {red }}^{p s}\right)^{0}$ resp. $\left(G_{\text {red }}^{s s}\right)^{0}$ is the Tannaka dual of $\lim _{X^{\prime}} \mathfrak{B}_{\text {red, } X^{\prime}}^{\text {ps resp. }}$ $\lim _{X^{\prime}} \mathcal{T}_{\text {red, } X^{\prime}}^{s s}$ shows the following: For $G$ as above, the group $\left(G^{0}\right)^{\text {ab }}$ is diagonalizable with character group

$$
X\left(\left(G^{0}\right)^{\mathrm{ab}}\right)=\underset{X^{\prime}}{\lim } \operatorname{Pic}_{X^{\prime}}^{0}\left(\mathbb{C}_{p}\right) .
$$

Note that the right hand group is torsionfree because line bundles of finite order become trivial in suitable finite étale coverings. This corresponds to the fact that $\left(G^{0}\right)^{\mathrm{ab}}$ is connected. We can therefore write as well:

$$
X\left(\left(G^{0}\right)^{\mathrm{ab}}\right)=\underset{X^{\prime}}{\lim }\left(\operatorname{Pic}_{X^{\prime}}^{0}\left(\mathbb{C}_{p}\right) / \text { tors }\right)
$$

and hence

$$
\left(G^{0}\right)^{\mathrm{ab}}={\underset{X^{\prime}}{\lim }}_{\operatorname{Hom}}\left(\mathrm{Pic}_{X^{\prime}}^{0}\left(\mathbb{C}_{p}\right) / \text { tors }, \mathbb{G}_{m, \mathbb{C}_{p}}\right)
$$

This is a pro-torus over $\mathbb{C}_{p}$.

In particular we have seen that $\left(G_{\text {red }}^{p s}\right)^{0}$ and $\left(G_{\text {red }}^{s s}\right)^{0}$ have the same maximal abelian quotient. Incidentally we may compare $\left(G, G^{0}\right)$ with $\left(G^{0}, G^{0}\right)$ :

General theory gives an exact sequence:

$$
1 \longrightarrow\left(G, G^{0}\right) /\left(G^{0}, G^{0}\right) \longrightarrow\left(G^{0}\right)^{\mathrm{ab}} \longrightarrow\left(G^{\mathrm{ab}}\right)^{0} \longrightarrow 1
$$

The sequence of character groups is

$$
1 \longrightarrow \operatorname{Pic}_{X}^{0}\left(\mathbb{C}_{p}\right) / \text { tors } \longrightarrow \underset{X^{\prime}}{\lim }\left(\operatorname{Pic}_{X^{\prime}}^{0}\left(\mathbb{C}_{p}\right) / \text { tors }\right) \longrightarrow X\left(\left(G, G^{0}\right) /\left(G^{0}, G^{0}\right)\right) \longrightarrow 1 .
$$


If $X$ is an elliptic curve, it follows that the first arrow is an isomorphism, so that $\left(G, G^{0}\right)=\left(G^{0}, G^{0}\right)$. If the genus satisfies $g(X) \geq 2$ then by the Riemann Hurwitz formula, the middle group is infinite dimensional and in particular $\left(G, G^{0}\right) /\left(G^{0}, G^{0}\right)$ contains a non-trivial pro-torus.

We proceed with some remarks on the structure of $G^{0}$ which follow from the general theory of reductive groups. Let $C$ be the neutral component of the center of $G^{0}$. Then we have

$$
G^{0}=C \cdot\left(G^{0}, G^{0}\right)
$$

Here $C$ is a pro-torus and $\left(G^{0}, G^{0}\right)$ is pro-semisimple. The projection $C \rightarrow\left(G^{0}\right)^{\text {ab }}$ is faithfully flat and its kernel is a commutative pro-finite groupscheme $H$. In the exact sequence:

$$
1 \longrightarrow X\left(\left(G^{0}\right)^{\mathrm{ab}}\right) \longrightarrow X(C) \longrightarrow X(H) \longrightarrow 1
$$

the group $X\left(\left(G^{0}\right)^{\mathrm{ab}}\right)$ is divisible because $\operatorname{Pic}_{X^{\prime}}^{0}\left(\mathbb{C}_{p}\right)$ is divisible. Since $X\left(\left(G^{0}\right)^{\mathrm{ab}}\right)$ is also torsionfree it is a $\mathbb{Q}$-vector space. The group $X(H)$ being torsion it follows that $X(C) \otimes \mathbb{Q}=X\left(\left(G^{0}\right)^{\mathrm{ab}}\right)$ canonically and $X(C) \cong X\left(\left(G^{0}\right)^{\mathrm{ab}}\right) \oplus X(H)$ non-canonically. It would be interesting to determine $X(H)$ for both $G=G_{\text {red }}^{p s}$ and $G_{\text {red }}^{s s}$.

We end with a remark on the Tannaka dual $G_{E}$ of the Tannaka subcategory of $\mathfrak{B}_{\text {red }}^{p s}$ generated by a vector bundle $E$ in $\mathfrak{B}_{\text {red }}^{p s}$. The group $G_{E}$ is a subgroup of $\mathbf{G L}_{E_{x}}$ the linear group over $\mathbb{C}_{p}$ of the $\mathbb{C}_{p}$-vector space $E_{x}$. It can be characterized as follows: The group $G_{E}\left(\mathbb{C}_{p}\right)$ consists of all $g$ in GL $\left(E_{x}\right)$ with $g\left(s_{x}\right)=s_{x}$ for all $n, m \geq 0$ and all sections $s$ in

$$
\Gamma\left(X_{\mathbb{C}_{p}},\left(E^{*}\right)^{\otimes n} \otimes E^{\otimes m}\right)=\operatorname{Hom}_{X_{\mathbb{C}_{p}}}\left(E^{\otimes n}, E^{\otimes m}\right) .
$$

Here $g\left(s_{x}\right)$ means the extension of $g$ to an automorphism $g$ of $\left(E_{x}^{*}\right)^{\otimes n} \otimes E_{x}^{\otimes m}$ applied to $s_{x}$.

Consider the representation attached to $E$ by theorem 8

$$
\rho_{E, x}: \pi_{1}(X, x) \longrightarrow \mathrm{GL}\left(E_{x}\right) .
$$

Its image is contained in $G_{E}\left(\mathbb{C}_{p}\right)$ because the functor $F \mapsto \rho_{F, x}$ on $\mathfrak{B}_{\text {red }}^{p s}$ is compatible with tensor products and duals and maps the trivial line bundle to the trivial representation. Hence $G_{E}$ contains the Zariski closure of $\operatorname{Im} \rho_{E, x}$ in $\mathbf{G} \mathbf{L}_{E_{x}}$. It follows from a result by Faltings $\left[\mathrm{F}\right.$. that the faithful functor $F \mapsto \rho_{F, x}$ is in fact fully faithful. If $\rho_{E, x}$ is also semisimple then a standard argument shows that $G_{E}$ is actually equal to the Zariski closure of $\operatorname{Im} \rho_{E, x}$. 


\section{References}

[At] M. Atiyah: Vector bundles over an elliptic curve. Proc. London Math. Soc. (3) 7 (1957), 414-452

[BLR] S. Bosch, W. Lütkebohmert, M. Raynaud: Néron models. Springer 1990

[DM] P. Deligne, J.S. Milne: Tannakian categories. Springer Lecture Notes 900

[DW1] C. Deninger, A. Werner: Line bundles and $p$-adic characters. To appear in a Texel Conference Proceedings

[DW2] C. Deninger, A. Werner: Vector bundles on $p$-adic curves and parallel transport. To appear in Ann. Scient. Ecole Norm. Sup.

[Du] L. Ducrohet: The action of the Frobenius map on rank 2 vector bundles over a supersingular genus 2 curve in characteristic 2. Preprint 2005. http://www . arXiv.math.AG/0504500

[EGAIV] A. Grothendieck, J. Dieudonné: Éléments de Géometrie Algébrique IV. Publ. Math. IHES 20 (1964), 24 (1965), 28 (1966), 32 (1967)

[F] G. Faltings: A p-adic Simpson correspondence. Preprint 2003

[Gie1] D. Giesecker: Stable vector bundles and the Frobenius morphism. Ann. scient. E.N.S. 6 (1973), 96-101

[Gie2] D. Giesecker: On a theorem of Bogomolov on Chern classes of stable bundles. Am. J. Math. 101 (1979), 79-85

[Ha] R. Hartshorne: Algebraic Geometry. Springer 1977

[HL] D. Huybrechts, M. Lehn: The geometry of moduli spaces of sheaves. Aspects of Mathematics, E31, Viehweg 1997

[JRXY] K. Joshi, S. Ramanan, E.Z. Xia, J.-K. Yu: On vector bundles destabilized by Frobenius pull-back. Preprint 2002. http://www . arXiv .math.AG/0208096

[LP] Y. Laszlo, C. Pauly: The action of the Frobenius map on rank 2 vector bundles in characteristic 2. Preprint 2004. http://www.arXiv.math.AG/0005044

[LS] H. Lange, U. Stuhler: Vektorbündel auf Kurven und Darstellungen der algebraischen Fundamentalgruppe. Math. Z. 156 (1977), 73-83

[Liu] Q. Liu: Algebraic Geometry and Arithmetic Curves. Oxford University Press 2002 
[N] M.V. Nori: On the representations of the fundamental group. Composition Math. 33 (1976), 29-41

[NS] M.S. Narasimhan, C.S. Seshadri: Stable and unitary vector bundles on a compact Riemann surface. Ann. Math. 82 (1965), 540-567

[RG] M. Raynaud, L. Gruson: Crières de platitude et de projectivité. Invent. Math. 13 (1971), 1-89

[S] J-P. Serre: Propriétés conjecturales des groupes de Galois motiviques et des représentations $l$-adiques. In: Jannsen et al. eds.: Motives. Proc. Symp. Pure Math. 55, 1, AMS 1994

[Si] C. Simpson: Higgs bundles and local systems. Inst. Hautes Études Sci. Publ. Math. 75 (1992), 5-92

[Ta] J. Tate: p-divisible groups. Proceedings of a Conference on local fields. Driebergen 1966, 158-183

[W] A. Weil: Généralisation des fonctions abéliennes. J. de Math. P. et App., (IX) 17 (1938), 47-87

Mathematisches Institut

Einsteinstr. 62

48149 Münster

Germany

deninger@math.uni-muenster.de
Fachbereich Mathematik

Pfaffenwaldring 57

70569 Stuttgart

Germany

werner@mathematik.uni-stuttgart.de 